\title{
Medición del nivel de estrés en el paciente
cáncer que acude al Servicio de Medicina Nuclear en el Hospital Regional de Alta Especialidad de Ciudad Victoria
}

\author{
Jorge Trejo-García ${ }^{1,2^{*}}$, Ana B. Rodríguez-Sánchez ${ }^{2}$ y Rosa A. Mata-Zúñiga ${ }^{2}$ \\ ${ }^{1}$ Medicina Nuclear, Hospital Regional de Alta Especialidad de Ciudad Victoria Bicentenario; 2Departamento de Docencia, Instituto de Ciencias y Estudios Superriores de
} Tamaulipas, Campus Victoria. Ciudad Victoria, Tamaulipas

\section{ABSTRACT}

Background: The psychological factor plays a contributory role in the predisposition to diseases. Stress is deffined as a set of alterations that occur in the body as a result of a demand on the individual in situations that exceed his or her resources. Patients with suspected cancer are required to attend the nuclear medicine area for complementary studies for diagnosis or treatment. Unfamiliarity of the patients with this area increases their levels of anxiety. Methods: A cross-sectional observational study was conducted in patients who attended the Nifear Medicine Service for the first time from October 2016 to June 2017. Patient participation was voluntary; inclusion criteria were an age older than 18 years and the previous diagnosis of cancer. Results: Mean age of patjents was $43 \pm 3$ years (range: 19-76 years). Acute stress was the most frequent type of stress in the analysis bygender, 10 (33.3\%) in women and $11(36.6 \%)$ in men, respectively. Conclusion: Stress is a disorder of high prevalence among patients who attend for the first time the Nuclear Medicine Service. (Hosp Med Clin Manag. 2019;12:165-8) Corresponding author: Jorge Trejo-García, hraev.jtrejo@gmail.com

Key words: Stress. Cancer. Nuclear Medicine.

\section{RESUMEN}

Introducción: El factor psicológico desempeña un papel contribuyente en la predisposición a enfermedades. El estrés se define como un conjunto de alteraciones que el organismo produce resultado de una exigencia sóbre el individuo en situaciones que exceden sus recursos. Los pacientes con sospecha de cáncer deben acudir al ắa de medicina nuclear para la realización de estudios complementarios para su diagnóstico o tratamiento. El desconocimiento de los pacientes acerca de esta área provoca una alteración en sus niveles de angustia. Métodos: 
una investigación observacional transversal en pacientes que acudieron por primera vez al Servicio de Medicina Nuclear de octubre de 2016 a junio de 2017. La participación de los pacientes fue voluntaria; se considerarof como criterios de inclusión una edad mayor a 18 años y el diagnóstico previo de cáncer. Resultados: La media dê edad de los pacientes fue de $43 \pm 3$ años (rango: 19-76 años). El estrés agudo fue el más frecuente en el análisis porr sexo, $10(33.3 \%)$ en mujeres y $11(36.6 \%)$ en hombres, respectivamente. Conclusión: El estrés es un trastorno@le alta prevalencia entre los pacientes que llegan por primera vez al Servicio de Medicina Nuclear.

Palabras clave: Estrés. Cáncer. Medicina nuclear.

\section{INTRODUCCIÓN}

Desde hace mucho tiempo se sabe que el factor psicológico desempeña un papel contribuyente en la predisposición, inicio o desarrollo de enfermedades. El estrés se define como un conjunto de alteraciones que se producen en el organismo como resultado de una exigencia sobre el individuo en situaciones que exceden sus recursos. Por lo tanto, el organismo se ve sumamente vulnerable ante ciertas enfermedades, provocando un efecto inmunosupresor. Se ha reconocido cada vez más que el estrés es un factor que puede reducir la calidad de vida de los pacientes. Existe incluso cierta evidencia que indica que el estrés extremo está asociado con peores resultados clínicos. Existen pautas clínicas disponibles para ayudar al personal médico y de enfermería a evaluar los grados de estrés y a ayudar a los pacientes a manejarlos. Por tal motivo, decidimos investigar cuál es el nivel de estrés en el paciente con cáncer que acude al Servicio de Medicina Nuclear. El estudio se justifica considerando que, de acuerdo con la Organización Mundial de la Salud, el estrés provoca el 25\% de los 75,000 infartos al año registrados en México. Hasta ahora, el $75 \%$ de los mexicanos padecen estrés, lo que coloca al país en el primer lugar mundial en esta categoría; le siguen China con un $73 \%$ y EE.UU. con un $59 \%{ }^{1}$.

La medicina nuclear se define como la especialidad médica que utiliza los radiofármacos para el diagnóstico, tratamiento e investigación de las enfermedades humanas. El estrés en el paciente que es atendido en el área de medicina nuclear está presente casi todo el tiempo, debido a la falta de conocimiento sobre esta nueva área médica². Cualquier suceso puede dar lugar a una respuesta emocional y generar estrés, por ejemplo, el nacimiento de un niño, el matrimonio, la muerte de un familiar o el diagnóstico de una enfermedad. No se trata necesariamente de eventos muy intensos, sino que es suficiente con que se acumulen durante largos periodos, y la manera en que la persona los o se enfrenta a ellos le afecta negativamente ${ }^{3}$. Actualmente, los pacientes con sospecha de cáncerđ̃deben acudir al área de medicina nuclear para la reatización de estudios complementarios para su diagnóśtico o incluso para su tratamiento. Debido al desconocimiento de los pacientes acerca de esta área médica, açudir a ella provoca una alteración en sus niveles de añgustia acumulados al estrés del proceso diagnóstico.

Existen tres tipos de estrés: el estrés agudo, que es la forma más común de estrés; el estrés agudo eppóndico, que se caracteriza por una sobreagitación prolong gada; y el estrés crónico ${ }^{4}$. En la actualidad, en el mupido los datos de las encuestas «condiciones de estrés" Fevelan que casi 150,000 hombres y 140,000 mujeres pâddecen estrés, ansiedad o depresión ${ }^{5}$. El estrés puede en@̣peorar los problemas de salud e incluso llevar a la ñiuerte; afrontar este problema, el cual actualmente aqueja a la mayor parte de la población mundial, es de vitaluimportancia para tener unas condiciones de vida óptimbas ${ }^{6}$.

Se cree que la población diagnosticada con căhncer y que es atendida en el área de medicina nuclear del Hospital Regional de Alta Especialidad de Ciudâd Victoria (HRAEV) presenta un alto nivel de estrés. Por ello, el objetivo del presente trabajo de investigación fue clasificar el nivel de estrés en pacientes con cânẹer que acuden al Servicio de Medicina Nuclear, examiّar las causas que llevan a este nivel de estrés, y ânalizar

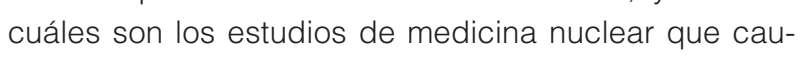
san con mayor frecuencia estrés para una posible intervención.

\section{MÉTODOS}

El tipo de estudio es no experimental-cuantitativo, con diseño observacional descriptivo de corte transversal, 
Tabla 1. Características clínicas y estudios realizados en el servicio de Medicina Nuclear

\begin{tabular}{lccc}
\hline \multicolumn{4}{l}{ Características clínicas y estudios realizados } \\
\hline $\begin{array}{l}\text { Edad (media } \pm \text { DE) } \\
\text { Sexo, } \mathbf{n}(\%)\end{array}$ & $43 \pm 3$ & & \\
$\quad$ Femenino & & & \\
\multicolumn{1}{l}{ Masculino } & $15(50)$ & & \\
\hline Gammagrama, $\mathbf{n ~ ( \% ) ~}$ & $\mathbf{n}=\mathbf{3 0}$ & Femenino & Masculino \\
& & $\mathbf{n}=\mathbf{1 5}$ & $\mathbf{n}=\mathbf{1 5}$ \\
\hline Óseo & $19(63.3)$ & $11(36.6)$ & $8(26.6)$ \\
Tiroideo & $6(20)$ & $4(13.3)$ & $2(6.6)$ \\
Renal & $2(6.7)$ & $1(3.3)$ & $1(3.3)$ \\
Hepatoesplénico & $2(6.7)$ & $1(3.3)$ & $1(3.3)$ \\
Mamario & $1(3.3)$ & $1(3.3)$ & $0(0)$ \\
\hline
\end{tabular}

DE: desviación estándar.

en pacientes que acudieron por primera vez al Servicio de Medicina Nuclear del HRAEV en el turno matutino en los meses de octubre de 2016 a junio de 2017. Los criterios de elegibilidad fueron ser mayores de edad, con diagnóstico previo de cáncer, con firma de consentimiento informado y participación voluntaria. Se excluyeron pacientes sin dominio del idioma español, pacientes que presentaban alguna deficiencia o discapacidad mental y mujeres embarazadas.

El instrumento utilizado fue un cuestionario autoaplicado que el paciente respondió brevemente dependiendo de su situación para poder identificar el nivel de estrés que tenía el paciente asociado a su diagnóstico y a la atención médica que recibía en el área de medicina nuclear. El instrumento aplicado fue el Test de Hamilton para medición del estrés, validado con un alfa de Cronbach de 0.918. Este cuestionario indica un estrés agudo en la puntuación < 80 puntos, y estrés agudo episódico cuando la puntuación es $>80$ puntos.

El procedimiento fue explicado a cada uno de los participantes y previo a la aplicación de la encuesta se pidió la firma del consentimiento informado. Esta investigación fue aprobada por el Comité de Ética del HRAEV.

El análisis estadístico se realizó con el software estadístico IBM SPSS ${ }^{\circledR}$ versión 21. Los datos se representan por medio de estadística descriptiva. Las variables cuantitativas se expresan mediante las medidas de tendencia central y dispersión, y las variables cualitativas, en frecuencias y proporciones.
Nivel de estrés de pacientes que acuden al Servicio de Medicina Nuclear en el HRAEV

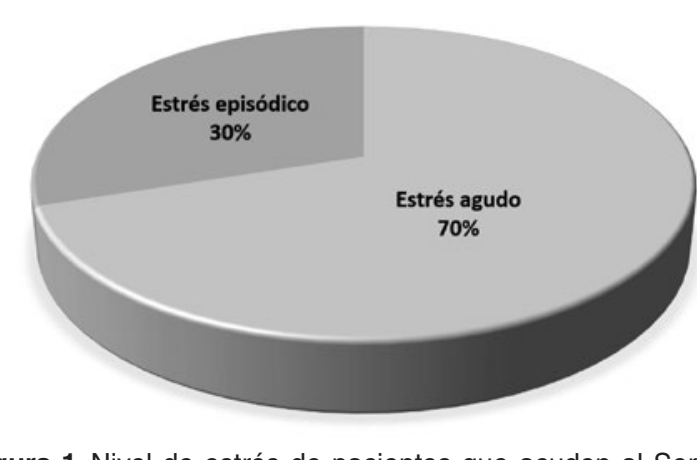

Figura 1. Nivel de estrés de pacientes que acuden al Servicio de Medicina Nuclear en el Hospital Regional de Alta Especialidad de Ciudad Victoria (HRAEV).

\section{RESULTADOS}

El test se aplicó en una muestra de 30 pacientešvoluntarios que acudieron al área de medicina nuclear del HRAEV. La media de edad de los pacientes fue de $43 \pm 3$ años (rango: 19-76 años). La distribución de la muestra por sexo fue de 15 (50\%) mujeres y 155 hombres, respectivamente.

El estudio diagnóstico más comúnmente realizădo fue el gammagrama óseo en 19 (63.3\%) pacientes, seguido de gammagrama tiroideo en $6(20 \%)$ pacientes. Entre las 15 mujeres que acudieron a recibir atención de medicina nuclear en el HRAEV, a 11 (36.6\%) ose les realizó gammagrama óseo, y a 8 (26.6\%) hombres se les realizó este mismo estudio. Los estudios de gammagrama menos frecuentes en el periodo de esta investigación fueron los renales, hepatoesplénicos y mamarios para ambos sexos (Tabla 1).

Una vez realizada la puntuación de los tests aplicados, se encontró que el estrés agudo fue el nivel deestrés que más predominó entre los pacientes estudiadōoss, con 21 casos (70\%) (Fig. 1). De esta manera, eÉEstrés agudo fue el más frecuente en el análisis pợ sexo, $10(33.3 \%)$ en mujeres y $11(36.6 \%)$ en hombrês, respectivamente. Por otra parte, $5(16.6 \%)$ mujeres y 4 (13.3\%) hombres presentaron estrés episódico.ð

\section{DISCUSIÓN}

Esta investigación centra la importancia de informar al paciente sobre el procedimiento que se sigue en las 
pruebas de diagnóstico y/o tratamiento, debido a que tener una información escasa puede provocar en el paciente un impacto emocional que repercute en su cooperación durante las maniobras de diagnóstico en el área de medicina nuclear, comprometiendo la eficiencia de los estudios. Así mismo, una investigación por un grupo de investigadores del Colegio de Enfermería de Sevilla menciona que es importante informar a los pacientes acerca de los exámenes y de los diferentes tratamientos a que serán sometidos a lo largo de la enfermedad durante todo el proceso terapéutico y no solo al inicio del diagnóstico, que es cuando hay un grado de estrés más elevado?

Otro estudio más reciente menciona que puede existir una relación entre el estrés y el desconocimiento sobre la dinámica de las pruebas diagnósticas en los pacientes que se someten a un estudio por primera vez en el Servicio de Medicina Nuclear ${ }^{8}$. Pifarré, et al. dicen que, en el contexto de pacientes con sospecha o diagnóstico de cáncer, la realización de pruebas diagnósticas desempeña un papel central de la actividad clínica y puede tener un elevado impacto psicológico?

Todos los pacientes de esta investigación acudieron por primera vez al Servicio de Medicina Nuclear a realizarse un estudio; 21 de 30 mostraron estrés agudo, que se manifiesta por presiones del pasado reciente y las exigencias y presiones anticipadas del futuro cercano, lo cual se relaciona con los resultados de Goldberg et al., en donde el estrés está relacionado con la dinámica de la prueba, los efectos secundarios y su resultado diagnóstico. Por ello, se considera importante la participación del personal de enfermería de medicina nuclear para brindar la información adecuada sobre el procedimiento de los estudios que se realizarán.

En México existe una elevada incidencia de cáncer entre la población y, como parte de su diagnóstico y tratamiento, el médico clínico solicita exámenes de gabinete especializados, como lo son los estudios de medicina nuclear. En esta investigación todos los pacientes que acudieron a Medicina Nuclear presentaban diagnóstico de cáncer; debido a su padecimiento y al desconocimiento del procedimiento, se encontró un nivel de estrés en los pacientes en donde el grado de estrés que más predominó fue el agudo. Por tanto, es conveniente que el personal de enfermería, que es el primer contacto con el paciente, le proporcione la información del procedimiento que se le va a realizar para bajar su nivel de estrés y así coopere durante sǜđestancia en el área de medicina nuclear. Lo anterior s్ se relaciona con los resultados obtenidos ${ }^{9}$ por Pifarré, हैt al.

\section{CONCLUSIONES}

El diagnóstico de una enfermedad supone enfrëntarse a una situación inesperada que genera importantes cambios en la vida de una persona. Esta investigación tuvo como propósito identificar el nivel de estrésıal cual se someten los pacientes cuando acuden al área de medicina nuclear.

El estrés es un trastorno de alta prevalencia entre cientes que llegan por primera vez al Servicio de Medicina Nuclear. Estos pacientes vienen totalmente desorientadados, con una carencia o deficiencia de información cögnitiva relacionada con la prueba que se les va a realizar. Este estrés viene provocado por una mala interpretaciợn de la información y la falta de comunicación por parte "đel personal de salud. Por lo anterior, es de gran impörtancia realizar los estudios de medicina nuclear adecŭada y oportunamente facilitados por la cooperación del páciente al disminuir su estrés con información de los diferentes procedimientos, mejorando los cuidados de enfermermería.

\section{FINANCIAMIENTO}

No fue requerido financiamiento para la realización del presente estudio.

\section{BIBLIOGRAFÍA}

1. Cáncer en cifras, Estadísticas INEGI 2010 [Internet]. México: Instituto Nacional de Estadística y Geografía [actualizado el 26 de marzo 2014]. Disp̄onible en: http://www.infocancer.org.mx/estadsticas-inegi-2014-con782i0.htmb

2. Soriano-Castrejón A, Martín-Comín J, García-Vicente AM. Medicína Nuclear en la práctica clínica. 2. ${ }^{a}$ Edición. Madrid: Grupo Aula Medica; 2015.

3. Estrés psicológico y el cáncer [Internet]. México: Instituto Nąional del Cáncer [actualizado el 24 de mayo 2015]. Disponible en: https://www cancer. gov/espanol/cancer/sobrellevar/sentimientos/hoja-informativa-estres

4. Ramos-Cejudo J, Cano-Vindel A. Tratamiento cognitivo-conducfual de un caso de trastorno por ansiedad generalizada: el componente meta cognitivo. Ansiedad y Estrés. 2008;14(2):305-19.

5. Belloch A, Sandín B, Ramos F. Manual de Psicopatología. Volumen II. Madrid: McGraw Hill; 2015.

6. Controlar el estrés [Internet]. Web Consultas Healthcare [actualizado el 26 de junio 2018]. Disponible en: http://www.webconsultas.combmente-yemociones/emociones-y-autoayuda/controlar-el-estres-772

7. Domínguez Moreno A, Alarcón Pato L. Relación de ayuda en pacięntes que se les va a realizar una PET-TAC. HYGIA. 2010;(74):38-43.

8. Goldberg DP, Hillier VF. A scaled version of the General Health-Questionnaire. Psychol Med. 1979;9(1):139-45.

9. Pifarre P, Simó M, Gispert JD, Pallarés MD, Plaza P, Martínez-Miralles E. Pruebas de diagnóstico por la imagen: ¿generan ansiedad? Rev Esp Med Nucl. 2011;30(6):346-50. 